\title{
Intelligent Agent-based Technique For Virtual Machine Resource Allocation For Energy-Efficient Cloud Data centres
}

\author{
OGECHUKWU M OKONOR, MO ADDA, ALEX GEGOV \\ School of Computing \\ University of Portsmouth \\ UNITED KINGDOM
}

\begin{abstract}
In recent years, cloud computing technology has emerged as a promising solution to many medium and small scale business enterprise. It leverages cost, encourages collaboration, and it is so flexible to use. Despite its attractive features, it still has some challenging issues such as power consumption rate. Although significant efforts have been devoted to optimising power usage through scheduling and virtualisation, it though remains a challenging problem. This paper introduces a new model on how to reduce the power consumption during live virtualisation and schedule based on intelligent mobile agent technology. The simulation results show that the proposed algorithm minimises power consumption used in the data centre at each stage by more than $30 \%$ compared to the current state-of-the-art techniques.
\end{abstract}

Key-words: energy efficiency, virtualisation, intelligent agent, cloud computing, data centre

Received: April 4, 2019. Revised: December 15, 2019. Accepted: January 23, 2020. Published: February 5, 2020.

\section{Introduction}

Data centres (DC) are structured electronic components which are responsible for its data processing (servers), its data communications (network component), and its data storage. These computing and networking components aid the processing, storing, and transmitting digital information in the information technology world. There has been a significant advancement in the rate at which people embrace technology. The adoption rate has become increasingly high due to recent innovations in smarter electronic gadgets. This digital transformation directly affects the DC performance, and inversely affects the energy consumption rate through its activities. Although some of the DC equipments were not originally designed to be active but has little or no heat resistor embedded in it. According to [1], real-time video streaming, online gaming, as well as mobile device are all on the increase. They contribute about $60 \%$ of all data traffic in cloud DC activities with a prediction of $80 \%$ rise by 2020 .

Furthermore, the service level agreement (SLA) binding the service providers and their clients DC runs $24 / 7$ all year round with a typical power density of $538-2153 \mathrm{w} / \mathrm{m}^{\wedge} 2$ [2]. The energy consumed by the DC is now so alarming.

In 2007, according to the report presented to the US congress shows that US data centre consumed about 61 billion kilowatt-hours in 2006, which is about $1.5 \%$ of the total U.S electricity consumption
[3]. The Garter report published three years later showed a further $0.5 \%$ increase [4].

In 2016, some of the data centre grant vendors claimed to be in control of the skyrocketing level of DC energy consumption. According to the U.S data centre energy usage report by Berkeley national laboratory [5], it claimed that due to the increasing awareness in data centre infrastructure operation, significant improvement has so far been seen in the energy efficiency of data centre structure. This is attributed to the adoption of hyperscale data centres which encourages a high level of virtualisation and reduces the use of physical servers. However, researchers and other industry experts have some doubts on this due to other research findings on energy efficiency involving cloud data centres.

While the US report estimates a static growth on its energy efficiency, the world is still struggling on the amount of energy used by the data centre systems which shows there is some level of hidden truth yet to be told by some of the vendors. According to the energy watch manger publication in 2019 [6], it shows that $4.7 \%$ of annual electricity usage globally is from the data centre.

Therefore, systematic observation has shown that a more dynamic method with intelligent operation should be the headway to finding a lasting remedy to high power usage in the cloud data centre. Before this study, the option of choosing the best energy consumption model suited to the needs of the data centre's high energy usage level has remained a 
challenging task due to the design complexity and heterogeneous nature of the cloud environment.

This paper is organised as follows: section 2 reviews the related works; section 3 proposed mathematical model for cloud DC system, section 4 the proposed intelligent agent technique for enhancing migration, section 5 is on the result analysis while section 6 is on conclusion and future work.

\section{Related Work}

The diurnal traffic of a data centre is complex and unpredictable to a certain level due to its dynamic nature - incoming traffic fluctuates between the peak and off-peak periods, though it varies based on so many factors. This has led to the massive deployment and over-provisioning of Cloud Computing resources to cope with traffic on a $24 / 7$ basis. It has been observed by Liu et al. [7] that the average load in data centres is about $30 \%$ of the resources, and this underscores the high level of resource overprovisioning. Therefore, the main aim of this paper is to reduce the number of inactive resources by using an intelligent and systematic approach. This process ensures that cloud data centre efficiency is the priority constraint, and the result shows that a substantial amount of energy is saved. It has also been shown that the line-card energy consumption in IP routers dominates the total energy used by the network to the tune of $43 \%$ of the total power of the router [8].

This research considers related work on virtualisation approach to power management data centre since the reliability and data centre performance depends significantly on it. Virtualization is the backbone of cloud computing resource pooling through the virtual machine monitor (VMM), also known as the hypervisor. Virtual machine (VM) hides the identity of the physical machine (PM) and then provide a virtualised environment for specific applications to run on. This related work considers mainly virtualisation consolidation by live migration and placement. Virtualisation technology is another essential approach used to leverage energy inefficiency in a cloud system; it enables the cloud provider to create many multiple virtual machine (VMS) instances on a single physical server, which in turn, improves the utilisation of resources. High energy consumption can be significantly reduced by switching off inactive nodes and also dynamically using live migration of VMs to reduce the activities in the physical server [9]. Tenna et al. also state that aggressive consolidation of VMs leads to performance degradation when there is a sharp increase in demand for resource usage, which this research work strongly disagrees with and will prove otherwise using an intelligent code. The resource requirement is essential to be understood and well managed because if the data centre resource requirement is not recognised and used appropriately, then it rapidly affects the response time, time-out, failure, and the service level agreement.

Prior work on power management using virtualisation on data centre is published by Nathuji and Schwan [10]. They designed the architecture of the data centre resource management system using intercontinental and local policies approach. The author proposed a technique that was able to manage the operating system (OS) power usage at a local and global level but failed to put into consideration automatic resource management at the global level in case of policy failure. Power usage management in cloud environment is an extensive study by [11]. In their work, power usage problem was seen as a sequential optimisation problem and was solved with the limited look-head control (LLC). They minimised power usage during active time and also maintained an excellent level of SLA. Verma et al. [12] went further to formulate power-aware dynamic placement as a bin packing problem with varying bin sizes. Their work was only valid for an online scenario with no consideration of SLA.

Beloglazov et al. (2011) [13] did many research works on energy efficiency in network and data centre. His work gave a good insight on how to improve VM consolidation by working on ways to detecting overloaded and underloaded hosts. They explored the selection of a new VM placement in an overloaded VM, which in turn improves the efficiency of the data centre. They proposed some of the several heuristics approaches for minimising energy during virtualisation such as the local regression method, minimum migration time and power-aware best-fit approach. However, they showed less implementation to justify their strategies on both physical machine and its network configuration.

The authors of [14] proposed an efficient resource management solution for consolidation that focused on reducing the energy consumption rate of the cloud data centre. They worked on the window moving average policy for determining overloaded PM and multi-criteria TOPSIS with prediction VM selection policy for VM selection from overloaded PMs. However, this has some limitations as well due to some congestion and bottlenecks during the 
implementation process, therefore not optimally minimising energy.

This paper focuses on three main issues: Mathematical formulation of the power consumption model, VM Prediction technique and Intelligent VM migration technique.

\section{Power model Formulation}

\subsection{Proposed Cloud DC Power Model}

A constructive look at the design of a modern data centre inspired this publication. For a better understanding of how a system operates and factors that contribute to its operation mechanism enables better management. The DC design content gave us a clearer picture of the essential factors to consider in the bid to minimise the power used in cloud DC. The bases of this power model formulation built from the ohm's law. Therefore, the energy used during each process, calculated as the amount of voltage remitted all over its resistor. According to our observation, data centre design comprises of these elements: processing, routing, connecting and virtual application elements. For clarity purpose, each element is also made up of specific components as shown below in vector form -

$$
\begin{aligned}
& \text { routing elements }\left(\mathrm{sw}_{1} \ldots \ldots \ldots \mathrm{sw}_{\mathrm{m}}\right) \\
& \text { connecting elements }\left(\mathrm{co}_{\mathrm{i}} \ldots \ldots \ldots \mathrm{co}_{\mathrm{c}}\right) \\
& \text { processing elements }\left(\operatorname{srv}_{\mathrm{i}} \ldots \ldots \ldots . \mathrm{srv}_{\mathrm{n}}\right)
\end{aligned}
$$

Virtual machine element for each server can be written in matrix form as -

$$
\left[\begin{array}{c}
\operatorname{srv} 1 \\
\operatorname{srv} 2 \\
\ldots \\
\text { srvn }
\end{array}\right] \text { contains }\left[\begin{array}{ccc}
\mathrm{vm}_{11} & \ldots . \mathrm{vm}_{\mathrm{n}} \\
\mathrm{vm}_{21} & \ldots . \mathrm{vm}_{2 \mathrm{~m}} \\
\ldots \ldots & \ldots \\
\mathrm{vm}_{\mathrm{n} 1} & \ldots . & . \mathrm{vm}_{\mathrm{nv}}
\end{array}\right]
$$

where

$$
\begin{aligned}
& \operatorname{srv}_{\mathrm{i}}: \text { the server } \\
& \mathrm{SW}_{1}: \text { the switch } \\
& \mathrm{v}_{\mathrm{m}}: \text { the virtual machine } \\
& \mathrm{Co}_{\mathrm{i}}: \text { the connector }
\end{aligned}
$$

Based on the cloud data centre design elements with its corresponding component as explained above, we then formulate the total amount of power consumed by the data centre at its standard operating rate and when an intelligent agent is involved. This brings us to calculating the complete power $\operatorname{DC}\left(P_{c d c}\right)$ consumed at a given time as:

$$
\begin{aligned}
P_{c d c}=\sum_{i=1}^{n} P_{s r v_{i}} & +\sum_{i=1}^{m} P_{s w_{i}}+\sum_{i=1}^{n} \sum_{j=1}^{v_{i}} P_{v m_{i j}} \\
& +\sum_{i=1}^{c} P_{c o_{i}}
\end{aligned}
$$

In this paper, the power consumed by connectors is ignored, and the power is proportional to the length of the cable and material type. Based on previous research work, the power consumed by the connecting element is, therefore, insignificant. Furthermore, Eqn 1 is reduced into smaller equations in order to enable a better understanding of this work. Switches form the basis of the interconnecting fabric that delivers task requests to the hosts for processing. Power consumption of a switch depends on the following: number of ports, port transmission rate, types of switch and the deployed cabling solution. Therefore, the power consumption model for switches becomes:

$$
P_{s w i}=P_{c h i}+\sum_{j=1}^{n_{l c}} P_{l c_{j}}+\sum_{j=1}^{n_{p i}} P_{r_{j}}
$$

where

$U_{i j}$ : Utilisation factor of an active port $j$ per switch $i$ $\mathrm{P}_{\text {chi }}$ : Power consumed by a switch $\mathrm{i}$ base on hardware chassis

$P_{l c}$ : Power consumed by the active line cards

$P_{r i}$ : Power consumed by the active port running at a transmission rate ' $r$ '

$$
P_{s w i}=P_{c h i}+\sum_{j=1}^{n_{l c}}\left(1-\beta_{j}\right) P_{l c_{j}}+\sum_{j=1}^{n_{p i}} P_{r_{j}} * U_{i j}
$$

$$
\beta_{i}=\left\{\begin{array}{l}
0 \quad \text { if a linecard is enable } \\
1 \quad \text { if a linecard is disable } \\
f_{i} \quad \text { if a switch is in standby }
\end{array}\right.
$$

$$
\begin{gathered}
P_{s w_{i}}=\hat{P}_{s w i}-\alpha_{i} \hat{P}_{s w i}-\left(1-\alpha_{i}\right) \hat{P}_{s w i} \\
\alpha_{i}=\left\{\begin{array}{cc}
0 & \text { if a switch is enable } \\
1 & \text { if a switch is disable } \\
f_{i} & \text { if } \text { a switch is in standby }
\end{array}\right.
\end{gathered}
$$

Computing server contributes immensely to the high energy consumption rate in the data centre. It will be ideal also to say that the energy consumption of a host server is proportional to the CPU utilization. Previous research has shown that idle server consumes two- 
thirds of its peak-load of the memory, dish and inputoutput resources running. Its energy model is now modified to represent the power consumption required for this work as follow:

$$
P_{\text {srvi }}=P_{c p u}+P_{\text {memory }}+P_{i o}
$$

Building on this, the energy model when the server is under some level of intelligent cost of power used by the server will then be written as -

$$
P_{s r v_{i}}=\hat{P}_{s}-\delta \hat{P}_{s}=(1-\delta) \hat{P}_{S}
$$

where $\delta_{i}=\left\{\begin{array}{c}0 \quad \text { if a server is enable } \\ 1 \quad \text { if a server is disable } \\ f_{i} \quad \text { if a server is on stand by }\end{array}\right.$

This is represented as $0 \leq \delta_{i} \leq 1, \mathrm{f}_{\mathrm{i}}$, the probability that a server goes on a standby mode.

Considering the power model of the Virtual Machine, the basic units of allocation of resource in the VM are taken into amount. Having

$$
V m=\{o p, r, \Delta t, h o s t\}
$$

where

$$
\begin{aligned}
& \text { op }=\text { operation of the } \mathrm{Vm} \\
& r=\text { the request scale of the } \mathrm{Vm} \\
& \Delta t=\text { the execution time of } \mathrm{Vm} \\
& \text { host }=\text { the hosted node of the } \mathrm{Vm}
\end{aligned}
$$

The total power for VM will then be -

$$
P_{V m}=\sum_{n=1}^{v i} P_{V m v i}
$$

Therefore, to evaluate the impact of an intelligent agent in the CDC, the total power consumed by the DC when an intelligent agent is in operation compared to Eqn 1 becomes:

$$
\begin{aligned}
\sum_{j=1}^{n}\left(1-\delta_{j}\right) P_{j}+ & \sum_{n=1}^{m} P_{c h i}+\sum_{j=1}^{n_{l c}}\left(1-\beta_{j}\right) P_{l c_{j}}+\sum_{j=1}^{n_{p i}} P_{r_{j}} \\
& * U_{i j}+\sum_{n=1}^{v i} P_{V m v i}
\end{aligned}
$$

\subsection{VM Prediction Technique}

Prediction is the ability to forecast the behavioural pattern of an incident which sometimes is under a probability. It can also have a very high chance of being true where the said system is highly studied and understood before creating its predictive tool. Considering the VM and user behaviour in the cloud data centre, it is essential to study and monitor its activities and the pattern at which it occurs. Therefore, this provides a reliable future resource processing technique that can manage and sustain efficient resource utilization rate in a dynamic cloud system scenario. To solve the prediction problem is to simply estimate the value of an output $(Y)$ from the sets of readily available inputs $(X)$ formulated as:

$$
\hat{Y}\left(T_{i}\right)=\hat{f}\left(X\left(T_{i}\right)\right.
$$

where

$\widehat{Y}\left(T_{i}\right)$ : predicted task value

$\hat{\mathrm{f}}\left(\mathrm{X}\left(\mathrm{T}_{\mathrm{i}}\right)\right.$ : estimated relation between tasks

Ismaeel S, Miri A [15] recorded that it is complicated to predict resource performance in cloud system due to its multi-resource nature. However, in this era of artificial intelligence and machine learning, it is simple and more straightforward to get the performance rate of a complex system like the cloud environment. The effectiveness of using an intelligent system to predict cloudlet real-time control, VM allocation, user behaviour, VM capacity planning and the rate at which energy is saved in the cloud system cannot be overemphasized. Using an intelligent agent approach overcomes the limitation of most research works encountered. An intelligent system helps to get a more accurate power consumption rate of the system by modelling and analysing the process power consumption pattern and the structures that affected the design.

Considering user $(U)$ submitting task $(T)$ at time $(t)$, the expectation $E\left(u_{i}\right)$ of each user is given by its probability $P\left(u_{i}\right)$, and the expectation $E\left(T_{i}\right)$ of a task send is given by its probability $P\left(T_{i}\right)$ conditioned to the probability of $P\left(u_{t}\right)$.

$$
E\left(u_{i}\right)=u_{i} P\left(u_{i}\right)+\left(E\left(T_{i}\right)=T_{i} P\left(T_{i}\right) / P\left(u_{t}\right)\right.
$$

Intelligent resource behavioural prediction pattern is important because it can be used as a guide to intelligent dynamic management decisions. In this paper, auto-scaling of VM resources with an intelligent agent is considered - this is able to scale down during underutilization and VM overloaded. The intelligent agent model (I.Agent) code when injected into the system, predict an intelligent workload pattern based on the resource requirement and its energy consumption rate. 


$$
\begin{aligned}
& D_{c_{L_{V}}}=I_{\text {agent }} *\left(\frac{1}{\left(1-U_{\text {mem }}\right)}\right)+I_{\text {agent }} * \\
& \left(\frac{1}{\left(1-U_{C P U}\right)}\right)+I_{\text {agent }} *\left(\frac{1}{\left(1-U_{\text {net }}\right)}\right)
\end{aligned}
$$

where

$$
\begin{aligned}
& U_{\text {mem }}: \text { memory utilization } \\
& U_{C P U}: C P U \text { utilization } \\
& U_{\text {net }}: \text { networkutilization }
\end{aligned}
$$

\section{Intelligent VM migration technique}

Performing VM migration is one of the vital approaches to minimizing power consumption in the CDC. VM migration is essential but also critical to resource management due to its complexity, that is, finding a trade-off between maintaining a high level of QoS and optimizing cloud resource utilization rate. It can easily lead to system downtime and violation of service level agreement. Therefore, significant understanding of VM migration and its migrations techniques are required for a comprehensive management of power usage in cloud design.

\subsection{Proposed Intelligent based migration technique}

According to previous research, different techniques were used to migrate VM in cloud system such as post copy, pre-copy and hybrid techniques. These techniques come with different setbacks - a high level of downtime for pre-copy [16] to migration, and taking longer time to migrate to a new host [17].

In this paper, an intelligence algorithm for choosing the best technique base on the cloud system requirement is proposed. An intelligent approach, in this case, calculates the amount of time it takes for a VM to migrate to a host, and it's power consumption cost.

Beloglazov and Buyya [13] laid a good foundation for understanding vital factors to consider while migrating a VM in the cloud environment. Hence an intelligent agent is used to determine an overloaded host and a particular VM for migration using a certain threshold. Using this approach, the power cost during migration can be calculated as follows$$
P_{\text {migration }}=P_{\text {source }}+P_{\text {destination }}=\left(\alpha_{\text {source }}+\right.
$$$$
\left.\alpha_{\text {destination }}\right) V_{\text {migration }}+\beta_{\text {source }}+\beta_{\text {destination }}
$$

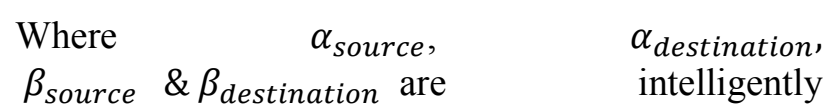
programmed to receive and send back different tasks.

To model the minimum power consumption cost function of this approach, we have -

$$
\min \sum\left(A C_{m g r}+C_{o p e r}\right) / C_{\text {uncert }}
$$

where

$A C_{m g r}$ : agent migration power consumption cost

$C_{\text {oper }}:$ the operational power consumption cost

$C_{\text {uncert }}:$ the uncertain events that affected the system transition activities

For this process to be understood, we define the underpin during VM migration, which is the dynamic scheduling of tasks based on available CPU capacity. SLA violation occurs in this process when the CPU demands exceeds its capacity. It is, therefore, essential to have some set conditions that guide the migration of resources and constraints attached to each state for the efficient operation of the VM. To determine the time it took by the VM to initiate a movement to minimize the total cost of operating the system, which consists of power cost and the SLAV with its constraints as shown in the cost function below:

$T C_{(v i, m k)}=\left\{\begin{array}{cr}(v i-m k) C_{p} & \text { if } m k<v i, v i-m k \geq \tau, \\ (v i-m k) C_{p}+2(m k-v i+\tau) C_{p}+(m k-v i) C_{v i} & \text { if } m k \leq v i, v i-m k \\ r C_{p}+(r-m k+v i) C_{p}+r C_{p} & \text { if } m k>v i,\end{array}\right.$

where:

$T C$ : the total cost function of operation which considers three cases of migration and violation under certain defined conditions

$v i$ : the violation history per transition

$m k$ : the migration curve

$r$ : the added time cause by event triggering or downtime 


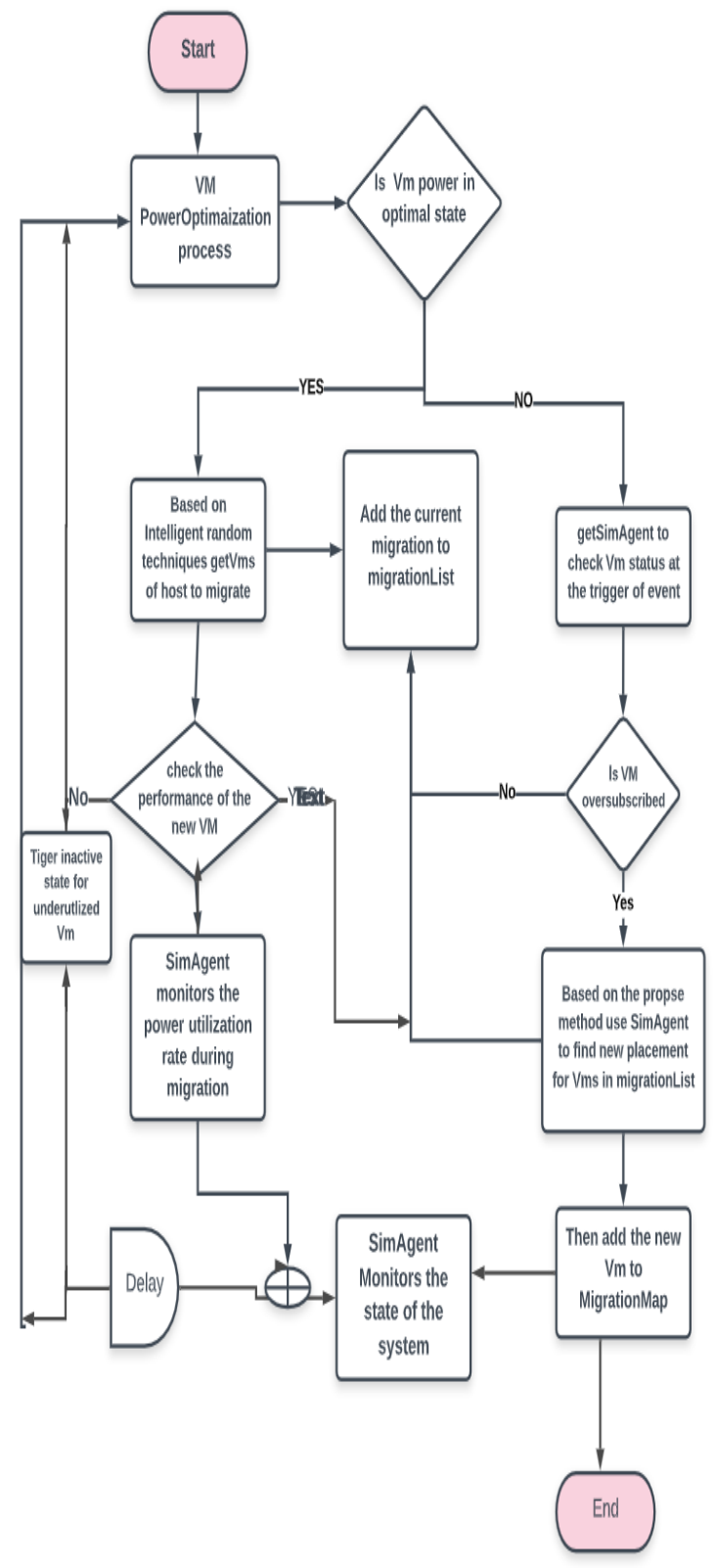

Figure 1: Intelligent flowchart for migrating VMs for power savings

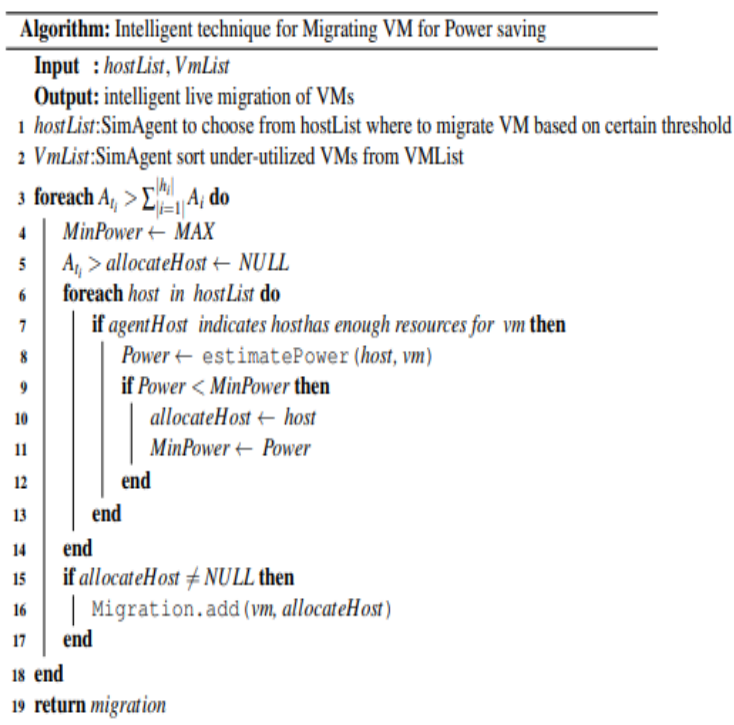

Figure 2: Intelligent algorithm for migrating VMs for power savings

\subsection{System Model}

In this section, the description of the system model used for this experiment is made clear. This paper considers a system with a large-scale data centre that has N-heterogeneous physical nodes, also known as the server where each of the servers has this attribute: CPU performance defined in millions of instructions per second (MIPS), amount of RAM and bandwidth. SLA is negotiated and agreed upon by the service provider and the user, depending on the QoS requirement. If there is any bridge of SLA, the service provider bears the responsibility. This setup consists of a software layer where the central and local manager function with an embedded agent in between. The global manager receives a request from users, sends it to the local network manager while the local agent autonomously migrates VM according to the specified requirement and threshold. 


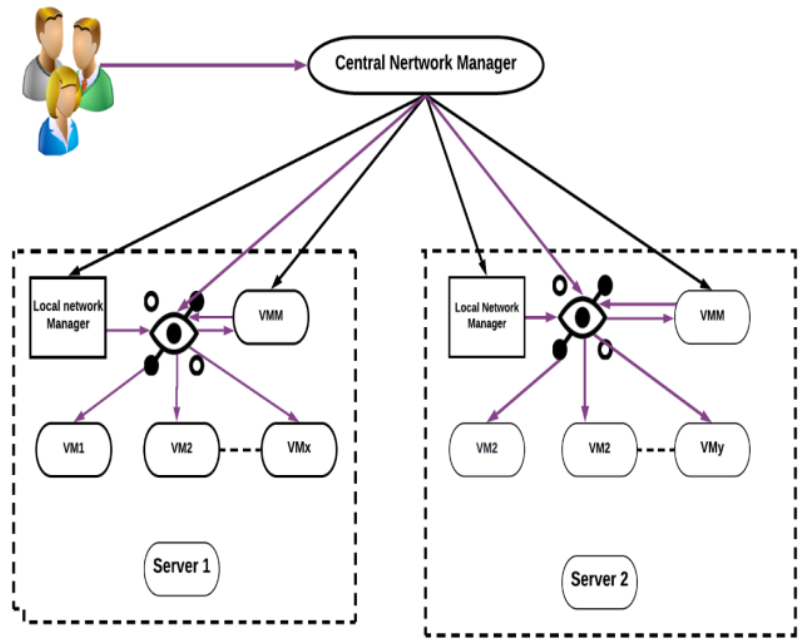

Figure 3: System Model

In this system model, the server has a multicore CPUs. Each multicore CPU has $\mathrm{K}$ cores, and each core has M MIPs as a single-core which make the CPU sum up its total capacity as $\mathrm{K}^{*} \mathrm{M}$ MIPS. Table 1 below gives a clear description of the server specification and configuration used in this research work.

\begin{tabular}{llrrr}
\multicolumn{6}{l}{ Table 1: Servers Specifications } & CPU Model & Cores & & Frequency(MHz) & RAM(GB) \\
\hline Server & Intel Xeon & 2 & 633 & 4 \\
HP Proliant G3 & Intel Xeon 3040 & 2 & 1,860 & 4 \\
HP Proliant G4 & Intel Xeon 3075 & 2 & 2660 & 4 \\
HP Proliant G5 & Intel Xeon 3470 & 4 & 2935 & 8 \\
IBM server X3250 & 12 & 3067 & 16 \\
\hline IBM server X3250 & $2^{*}$ Intel 5675 & 12 & &
\end{tabular}

\subsection{Experiment setup}

In this research work, Cloudsim toolkit is used because it is an extensible open-source toolkit with built-in capability to compare power-aware algorithm with its performance metric. Cloudsim also models the real-time of the federated cloud environment and is now the most acceptable platform for conducting cloud base simulation. It features support virtualisation engine which enables the creation of adequate management of independent, multiple and virtualised services on the data centre host.

The experiment was set up for 10 days with data from CoMon project [18] with more than 600 heterogeneous physical machines, monitoring infrastructure from PlanetLab. The data contains the CPU utilisation with 5-minute interval recording from more than $1000 \mathrm{VMs}$. The workload traces is an infrastructure-as-a-service cloud model dataset, which is ideal for this piece of work. It also modelled Amazon elastic compute cloud (EC) and IBM specification. We used a random workload with random scheduling technique with some intelligent embedded message accessing all data centre. In each scenario, each VM runs a variable workload with agent monitoring and intelligent messaging technology to ensure efficiency. Table 2 shows the power consumption of the chosen server specification in Table 1 based on a 90\% CPU utilization rate using $90 \%$ utilisation rate leverage automatic downtime due to overload.

\begin{tabular}{|c|c|c|c|c|c|c|c|c|c|c|}
\hline Table 2 & Pow & nsu & ion & ervers & & & & & & \\
\hline Server & idle & $10 \%$ & $20 \%$ & $30 \%$ & $40 \%$ & $50 \%$ & $60 \%$ & $70 \%$ & $80 \%$ & $90 \%$ \\
\hline $\begin{array}{l}\text { HP ProLiant } \\
\text { G3 }\end{array}$ & 105 & 112 & 118 & 125 & 131 & 137 & 147 & 153 & 157 & 164 \\
\hline $\begin{array}{l}\text { HP ProLiant } \\
\text { G4 }\end{array}$ & 86 & 89.4 & 92.6 & 96 & 99.5 & 102 & 106 & 108 & 112 & 114 \\
\hline $\begin{array}{l}\text { HP ProLiant } \\
\text { G5 }\end{array}$ & 93.7 & 97 & 101 & 105 & 110 & 116 & 121 & 125 & 129 & 133 \\
\hline $\begin{array}{l}\text { IBM Server } \\
\text { X3250 }\end{array}$ & 41.6 & 46.7 & 52.3 & 57.9 & 65.4 & 73 & 80.7 & 89.5 & 99.6 & 105 \\
\hline $\begin{array}{l}\text { IBM Server } \\
\text { X3250 }\end{array}$ & 66 & 107 & 102 & 131 & 143 & 156 & 173 & 191 & 211 & 229 \\
\hline
\end{tabular}

For any performance in the system to be valid, it must consider the SLA violation (VSLA) metric. We consider for each approach the SLA violation that occurs during the process and the Performance degradation Management level (PDML) of each transaction caused by the system. We then model as follows -

1: $\quad$ SLA violation per active server(SLATASv): where CPU utilization reached $100 \%$.

$S L A T A S v=\frac{1}{N_{s v}} \sum_{i=1}^{N_{s v}} \frac{T_{s i}}{T_{\alpha i}}$

where

$N_{s v}$ : number of servers

$T_{s i}$ : total time it took the server to reach $100 \%$ utilisation

$T_{\alpha i}$ : active server per time

2: $\quad$ Performance degradation management level is observing the live migration of VM from one server to the other without obstruction. Degradation can be affected by many application behavioural factors. The estimated performance degradation management level on the CPU at $10 \%$ utilization rate is as follows:

$$
P D M L=\frac{1}{N_{V m s}} \sum_{j=1}^{N_{v m}} \frac{C_{d j}}{C_{r j}}
$$


where

$N_{V m s}:$ is the number of vms

$C_{d j}$ : estimated as $10 \% \mathrm{cpu}$ utilization of vmj in all migration

\section{$C_{r j}:$ total cpu requested by $\mathrm{vmj}$}

Hence, the equation for formulating VSLA is then calculated by combining the two metrics in Eqn 16 and Eqn 17.

$$
V S L A=S L A T A S v * P D M L
$$

Furthermore, for clarity purpose, we defined the characteristic, Mips and workload data in Table 3.

Table 3

$$
\text { Virtual Machine (EC2) }
$$

\begin{tabular}{lrr}
\hline VM Type & CPU(MIPS) & RAM(GB) \\
\hline High-Memory Extra Large & 3,000 & 6,000 \\
High-CPU Medium & 2,500 & 850 \\
Extra Large Instance & 2,000 & 3750 \\
Small Instance & 1,000 & 1,700 \\
Micro Instance & 500 & 633 \\
\end{tabular}

Table 4 represents the conducted parameters of workload calculated over 10 days of the experiment.

\begin{tabular}{lrrr} 
Table 4 & \multicolumn{3}{l}{ Workload data characteristics. } \\
\hline Data & Number of VMs & Mean(\%) & SD(\%) \\
\hline $03 / 03 / 2011$ & 1052 & 12.31 & 17.09 \\
$06 / 03 / 2011$ & 898 & 11.44 & 16.83 \\
$09 / 03 / 2011$ & 1061 & 10.7 & 15.57 \\
$22 / 03 / 2011$ & 1516 & 9.26 & 12.78 \\
$25 / 03 / 2011$ & 1078 & 10.56 & 14.14 \\
$03 / 04 / 2011$ & 1463 & 12.39 & 16.55 \\
$09 / 04 / 2011$ & 1358 & 11.12 & 15.09 \\
$11 / 04 / 2011$ & 1233 & 11.56 & 15.07 \\
$12 / 04 / 2011$ & 1054 & 11.54 & 15.15 \\
$20 / 04 / 2011$ & 1033 & 10.43 & 15.21 \\
\hline
\end{tabular}

\section{Result Evaluation}

The obtained result analysis showed that this approach was able to save a significant amount of power during the processing time compared to other research works. Figure 4 shows the amount of energy saved at each virtual machine migration period using our algorithm. It is evident that at each traffic metric stage a substantial amount was saved
Figure 4: amount of energy saved at each virtual machine migration period

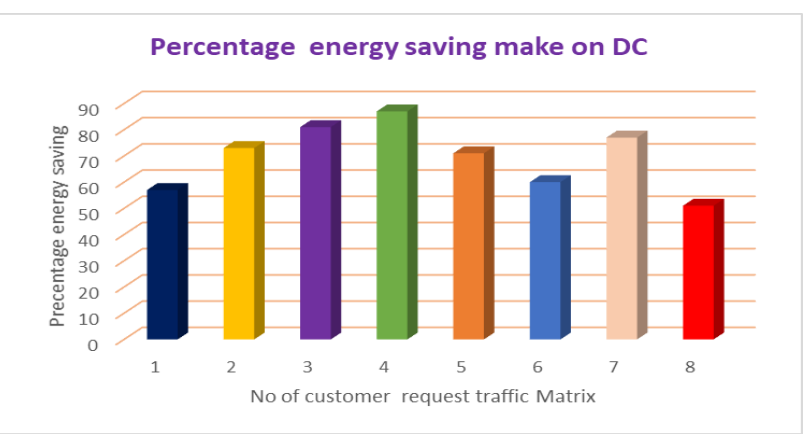

Furthermore, from the graphical result in Figure 5, it showed that when an intelligent agent code was introduced into the already existing dynamic threshold approach, it checked the migration process more efficiently and had minimal SLA violation. However, we did not include it as a graph in this paper.

Figure 5: Comparison analysis of three different VM threshold

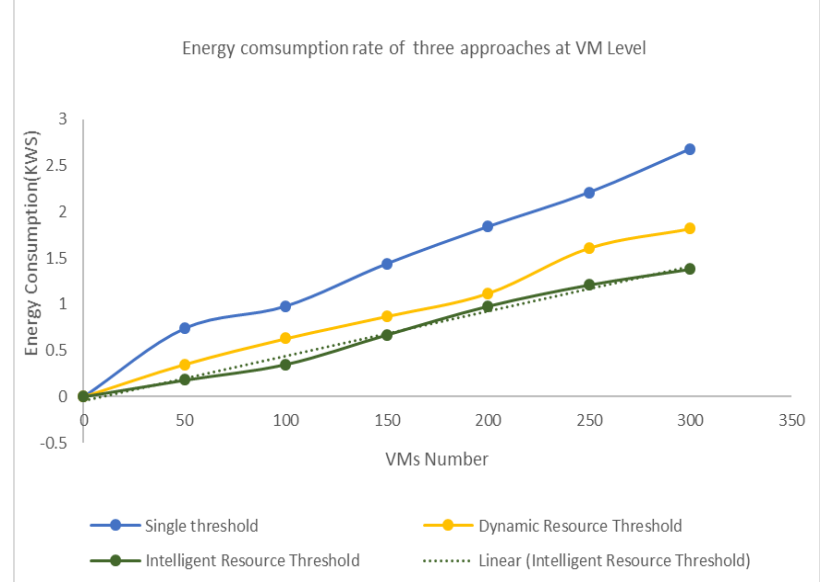

Continuing with result evaluation, we also examined the impact of our propose algorithm to the other existing ones used on cloudsim environment which are known to be the linear regression (LR), mean absolute deviation (MAD) and interquartile range(IQR). According to Figure 6 graphical representation, it shows that the intelligent agent(I.Agent) approach consumed less power than the other scheduling approaches in the real workload traces. Figure 6 depicts that it reduced the amount of power it consumed by up to $26.9 \%$ with desirable system performance from experimental setup traces. Moreover, it achieved a significant reduction of $68.9 \%, 17.4 \%, 14.6 \%$, and $24.1 \%$ in power consumption when compared to LR, THR, MAD, and IQR methods. 
Figure 6: Power consumed by I.Agent method compared to other scheduling approaches

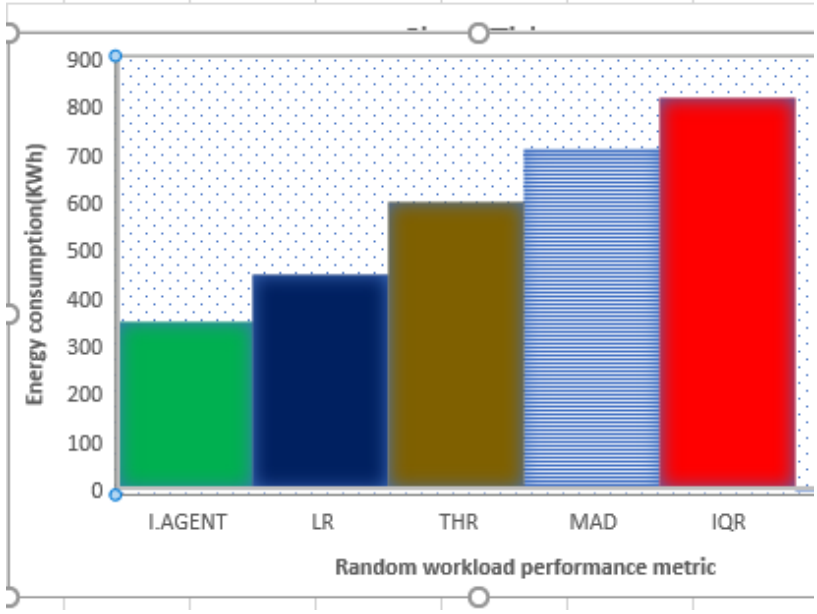

\section{Conclusion and Future work}

As cloud service providers continue to invest in innovative technology that can leverage the highpower consumption in a cloud data centre. Our proposed approach is one of its kind, that can intelligently minimise power with minimal downtime and therefore, do not encourage performance degradation. Our work brought the tradeoff between power efficiency and system performance. When compares to the existing dynamic VM consolidation methods, our proposed method provides more optimal energy-efficient solution.

Future work will model each level of SLA violation that occurred at each stage of this work. It will also test this approach on switch performance in cloudsim kit while enhancing this performance presentation.

\section{References:}

[1] Li B., et al.:EnaCloud: an energy-saving application live placement approach for cloud computing environments, Proc of international conf on cloud computing(2009)

[2] Oro E, Depoorter V, Garcia A, Salom J. Energy efficiency and renewable and energy integration in data centres. Strategies and modelling Review. Renewable and Sustainable Energy Review 2015;429-445

[3] Brown, R.: Report to congress on server and data centre energy efficiency public law 109-431," U.S. Environ. Protection Agency, Washington, DC, USA (2007).

[4] Gartner Press Release April 26, 2007, Gartner Estimates ICT Industry Accounts for 2 percent of global CO2Emissions.http://www.gartner.com/it/ page.jsp?id=503867

[5] OgechukwuOkonor, Mo Adda" Intelligent Approach to Minimizing Power Consumption in Cloud-Based System Collecting Sensor data and Monitoring the Status of Powered Wheelchair" Intelligent system conference 1, 2019.

[6] OgechukwuOkonor, Mo Adda" Power Optimisation Model for Leveraging Cloud System", IEEE Conference, 2019.

[7] Liu, J., Zhao, F., Liu, X., He, W.: Challenges Towards Elastic Power Management in Internet Data Centres. Proc 2nd international workshop on cyber-physical systems (WCPS), in conjunction with ICDCS Montreal, Canada, (2009

[8] Zhang, Q., Cheng, L., Boutaba, R.: Cloud computing: state-of-the-art and research challenges" Published online: The Brazilian Computer Society (2010).

[9] Tenna Mathew, KC Sekaran, J Jose -"Study and analysis of various task scheduling algorithm in the cloud computing environment". $2014 \quad$ International conference ..., 2014 - ieeexplore.ieee.org

[10]Nathuji R, Schwan K. Virtualpower: coordinated power Management in virtualized enterprise systems ACM SIGOPS Operating Systems Review 2007;41(6):265-278. 
[11]Kusic D, Kephart J O, Hanson JE, Kandasamy N, Jiang G. Power and Performance management in virtualised computing environments via lookahead control. Cluster computing 2009,12(1):1-15.

[12] Verma A, Ahuja P, NeogiA.pMapper" power and migration cost aware application placement in virtualized systems proceedings of the 9th ACM/IFIP/USENIX international conference on middleware, Springer, Leuven, Belgium, 2008;243-264.

[13] Beloglazov," optimal Online Deterministic Algorithm and Adaptive Heuristics for Energy and performance efficient dynamic consolidation of the virtual machine in the cloud Data centre" Volume 24.

[14] ArianyaE, Taherir H, Sharifian S(2015) Novel Energy and SLA efficient resource management heuristics for consolidation of virtual machines in cloud data centres.Comput Elect Eng.

[15] Ismaeel S, Miri A(2015) Using ELM technique to predict data centre $\mathrm{VM}$ request. In proceeding of the 2 nd IEEE international conference on cybersecurity and cloud computing(CS Cloud 2015).IEEE, New York,pp80-86.

[16] Hu B, Lei Z, Lei Y, Xu D(2011) A time series based pre-copy approach for live migration of virtual machines. In: Parallel and Distributed System(ICPADS)2011 IEEE 17th international conference on. IEEE, pp 947-952.

[17] Cui, J., Liu, S.f, Zeng, B., Xie, N.M: A novel grey forecasting model and its optimization. Applied Mathematical Modelling 37(6), 4399-4406(2013).

[18] SPEc power benchmarks, Standard Performance EvaluationCorporation. http://www.spec.org/benchmarks.html\#pow er 